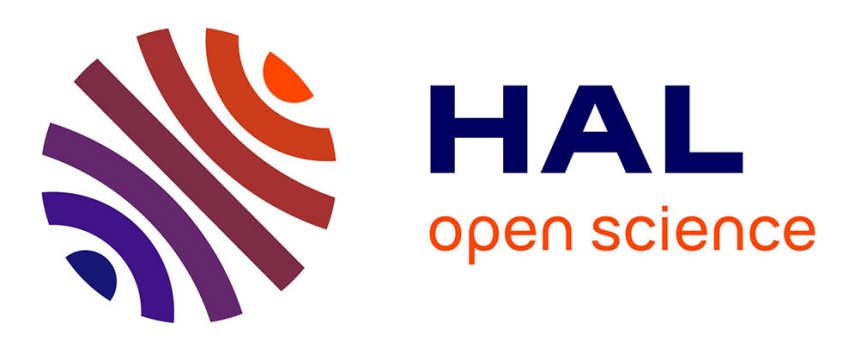

\title{
Aficionados et opposants à la tauromachie. Les formes plurielles de la civilisation
}

Christophe Traïni

\section{To cite this version:}

Christophe Traïni. Aficionados et opposants à la tauromachie. Les formes plurielles de la civilisation. Politix, 2003. halshs-02557476

\section{HAL Id: halshs-02557476 \\ https://shs.hal.science/halshs-02557476}

Submitted on 28 Apr 2020

HAL is a multi-disciplinary open access archive for the deposit and dissemination of scientific research documents, whether they are published or not. The documents may come from teaching and research institutions in France or abroad, or from public or private research centers.
L'archive ouverte pluridisciplinaire HAL, est destinée au dépôt et à la diffusion de documents scientifiques de niveau recherche, publiés ou non, émanant des établissements d'enseignement et de recherche français ou étrangers, des laboratoires publics ou privés. 


\section{Aficionados et opposants à la tauromachie.}

Les formes plurielles de la civilisation.

Christophe TRAÏNI

Depuis quelques temps déjà, la violence faite aux animaux alimente de manière de plus en plus ostensible l'activisme d'entrepreneurs de causes soucieux de promouvoir le «Bien-être animal ». L'opposition à la tauromachie espagnole constitue un cas remarquable de ces engagements militants qui entendent s'insurger contre les attitudes déplorables que l'homme peut nourrir à l'égard de l'animal. Selon ses promoteurs, en effet, le combat contre la corrida serait d'autant plus impérieux et emblématique que cette dernière ferait « obstacle à l'amélioration du sort de tous les animaux » $(\mathrm{d} 7)^{1}$ :

La corrida est l'affirmation brutale que l'animal, même domestique, n'a aucun droit et que, contre lui, l'homme peut tout se permettre, y compris de le torturer à mort pour se distraire (d9).

L'Alliance pour la suppression des corridas, régie par la loi 1901, a pour objet de protéger et de défendre les droits à la vie, à la liberté, au bien-être et au respect de tous les animaux et des taureaux de corrida en particulier (d4).

L'animal dans la Constitution Européenne. Contribution de la Fédération de liaisons anti-corrida. Article 1 : Nul ne peut se prévaloir de l'exception culturelle sur le territoire européen pour favoriser le maintien ou l'extension de pratiques violentes et de discriminations arbitraires à l'endroit du monde animal, notamment dans le divertissement (d6).

Comme on peut le voir ici, les opposants à la tauromachie se réclament de principes éthiques qui dépassent très largement la seule question du sort réservé au taureau dans l'arène. De fait, leurs revendications militantes pourraient faire l'objet d'une énième analyse philosophique des tenants et aboutissants axiologiques qui les sous-tendent. La souffrance animale est-elle compatible avec l'éthique humaniste? En quoi des prémisses, par exemple, utilitaristes commandent-ils la réprobation de cette souffrance suscitée par l'homme ? Faut-il considérer que les animaux ont des « droits » ou que les hommes ont des « devoirs » à leur égard² ? Dans une telle optique, le souci de symétrie exigerait sans doute que l'on examine également la cohérence des principes que les partisans de la tauromachie évoquent pour justifier la pratique : manifestation d'une esthétique ineffable ou d'un culte immémorial du taureau, spécificité irréductible des cultures populaires méridionales, prégnance d'un rituel relevant d'une anthropologie fondamentale de l'homme à michemin entre les besoins irrésistibles de «sacré » et les lois d'airain de la psychanalyse. Aussi stimulantes soient-elles, ces théories en usage — hostiles ou favorables à la corrida — risquent fort d'entraîner et de perdre l'observateur dans les méandres d'interminables controverses. Le plus regrettable, dès lors, serait sans doute de succomber à un biais cognitiviste selon lequel la passion ou l'aversion à l'égard de la tauromachie relèverait exclusivement des capacités logiques d'argumentation des acteurs sociaux. A l'encontre d'une telle perspective, cet article s'efforcera

1 On trouvera à la fin de l'article les références des documents cités $(\mathrm{d} 1, \mathrm{~d} 2, \ldots)$. Le corpus en question est presque exclusivement constitué de textes militants produits par l'Alliance pour la suppression des corridas et la Fédération de liaisons anti-corrida. Cette analyse purement textuelle se justifie d'autant plus qu'elle sera ici entièrement subordonnée à l'examen des représentations du statut de l'animal. Il ne sera donc pas question ici d'une étude visant à rendre compte des conversions militantes, des modes d'action et d'organisation, des formes de recrutement, propres aux associations anti-corrida qui pourront faire l'objet d'autres articles.

2 Sur les controverses philosophiques concernant ces questions, on pourra consulter Goffi (J-Y.), Le philosophe et ses animaux. Du statut éthique de l'animal, Editions Jacqueline Chambon, Nîmes, 1994. Ferry (L.), Le nouvel ordre écologique. L'arbre, l'animal et l'homme, Grasset, 1992. Ost (F.), La nature hors la loi. L'écologie à l'épreuve du droit, notamment le chapitre cinq, Paris, la Découverte, 2003. 
d'expliciter dans quelle mesure les différends relatifs à la tauromachie sont loin de se réduire à des débats philosophiques sur le statut éthique de l'animal. Nous sommes convaincus, en effet, que le rapport à l'animal - qui commande l'attitude à l'égard de la corrida - repose sur des formes d'économies émotionnelles qui préexistent, pour ainsi dire, à toute tentative de rationalisation et de justification par des principes. L'enjeu est donc bien de se départir d'une coupure rationnel/émotionnel souvent trompeuse afin de mettre en exergue ce que les modes de perception du taureau de combat doivent avant tout à des formes de socialisation au sein de configurations sociales incluant des logiques psychologiques ${ }^{3}$. Dans cette optique, l'œuvre de Norbert Élias semble nous offrir des outils particulièrement aptes à interroger la sociogenèse et la psychogenèse des adhésions ou des rejets passionnels que le spectacle tauromachique suscite avec une intensité souvent peu commune. Le recours au cadre théorique du sociologue allemand nous incitera notamment à relever certaines homologies que partagent - contre toute apparence - la passion pour la tauromachie et la compassion pour ses "victimes » animales. Ce faisant, nous espérons rendre un peu plus intelligible les fondements de l'incompréhension réciproque qui divise les adeptes et les détracteurs de la mise à mort spectaculaire des taureaux. Comme on pourra le constater, l'ampleur de cette incompréhension apparaît particulièrement adapté pour démontrer dans quelle mesure le processus de «civilisation» théorisé par Norbert Élias ne devrait être conçu autrement que comme un terme générique recouvrant de multiples modalités plus ou moins convergentes. Une juste compréhension et un bon usage de l'oeuvre du sociologue allemand, en effet, exigent que la plus grande attention soit accordée, non pas à une supposée " civilisation » univoque, mais bien plutôt à la pluralité, aux dissensions, aux tensions et conflits que ses diverses manifestations impliquent ${ }^{4}$.

\section{La civilisation des mœurs : tableau idéal-typique}

L'oeuvre d'Élias a suffisamment été discutée ces dernières années ${ }^{5}$ pour que nous puissions nous contenter ici d'un rapide rappel des ses principaux enseignements. Comme chacun sait, le sociologue allemand préconise de resituer les faits étudiés au sein d'un schéma général des évolutions qui les ont précédés. Loin de vouloir dévoiler — à l'instar d'un Fukuyama pressé — un sens inéluctable et unilinéaire de l'histoire, le sociologue allemand s'efforce avant tout de mettre en lumière l'interdépendance de phénomènes trop souvent pensés comme des objets radicalement distincts. D'une part, la centralisation du pouvoir et la monopolisation de la violence légitime par des institutions étatiques fortement spécialisées, objet privilégié d'une macrosociologie trop souvent aveugle aux comportements individuels. D'autre part, l'évolution de phénomènes trop aisément rabattus sur une dimension exclusivement psychologique - rapport à soi et à autrui, convenances extérieures du corps, sensibilités et manifestation des émotions - que Norbert Élias place plus particulièrement au cœur de ses analyses de la "civilisation des moeurs »" Ce sont plus précisément les éléments qui caractérisent cette notion qu'il importe, pour la suite de notre propos, de rappeler à grands traits. Afin d'éviter une lecture excessivement évolutionniste - qui, il est vrai, pourrait être alimentée par certains textes ambigus de Norbert Élias - nous nous en tiendrons ici une lecture a minima des grandes tendances historiques théorisées par l'auteur du Procès de la civilisation. Il faut entendre, par là, que le schéma général décrit par le sociologue allemand doit simplement être rapporté à des domaines bien circonscrits — tel par exemple, le rapport à l'animal,

\footnotetext{
3 Sur la pertinence heuristique des approches de ce type, voir Braud (P.), L'émotion en politique. Problèmes d'analyse, Paris, PFNSP, 1996.

4 Dès le premier chapitre de La civilisation des mours, Norbert Élias propose une analyse comparative des manifestations différentielles de la «civilisation » en France et en Allemagne qui interdit d'emblée de l'envisager comme un phénomène unique et homogène.

5 Voir, par exemple, Garrigou (A.) et Lacroix (B.), Norbert Élias, la politique et l'histoire, Paris, La Découverte, 1997. Heinich (N.), La sociologie de Norbert Élias, Paris, La Découverte \& Syros, 1997. Haroche (C.), « Retenue dans les mœurs et maîtrise de la violence politique. La thèse de Norbert Élias », dans Braud (P.), La violence politique dans les démocraties européennes occidentales, Paris, L’Harmattan, 1993. Déchaux (J.-H.), « Sur le concept de configuration : quelques failles dans la sociologie de Norbert Élias », Cahiers internationaux de sociologie, volume 99, 1995. 6 Élias (N.), La civilisation des mœurs, Paris, Calmann-Lévy, 1973.
} 
à la tauromachie ou à la chasse ${ }^{7}$ — et ce afin d'expliciter pourquoi certaines conduites tendent à provoquer des conflits de valeur entre des groupes d'individus différemment socialisés.

L'un des indices majeurs de la civilisation des mœurs transparaît à travers l'abaissement croissant, à partir de la Renaissance, du seuil de tolérance à l'égard de l'agressivité. La " pacification » de la société par un pouvoir central revendiquant le monopole de la violence, ainsi que la « curialisation » d'une aristocratie autrefois guerrière, ont favorisé la dépréciation des affrontements physiques et sanguinaires. Les classes supérieures, en effet, tendirent à se soumettre à une étiquette dictant des règles de civilité de plus en plus sophistiquées et raffinées. L'expression des émotions emprunta des voies toujours plus contraintes par l'exigence de retenue et de contrôle des dispositions les plus combatives. La civilisation des mœurs dévalorisa ainsi la férocité et la brutalité des conduites et exalta a contrario la mesure, la pondération, le tact et les prévenances à l'égard du prochain. La pudeur notamment se développa du fait de la crainte d'une vulnérabilité face à des agressions extérieures paraissant de plus en plus préoccupantes. A cela s'ajouta inextricablement la dépréciation de la visibilité des fonctions naturelles. L'homme civilisé, en effet, doit savoir «se tenir » et dissimuler au regard d'autrui le fait de se moucher, de cracher, de déféquer, et de faire état de ses envies (notamment sexuelles). Dans un tel contexte, les activités liées au sang ont été plus particulièrement déconsidérées. Dans le même ordre d'idée, et à la suite des travaux de l'historien Philippe Ariès, Norbert Élias ${ }^{8}$ souligne comment l'intensification de la pudeur a entraîné avec elle une propension croissante à percevoir la mort comme une réalité « sale et inconvenante ${ }^{9}$.

Pour résumer très schématiquement, les hommes civilisés n'aiment ni la violence physique, ni les désirs et émotions exprimés sans retenue, et tendent, par ailleurs, à dissimuler toute activité liée au sang et à la mort. Les formes de sensibilité qui les caractérisent les incitent donc à se plier à une autocontrainte fortement intériorisée visant à maîtriser tout affect contraire aux exigences de la civilisation. Un tel mécanisme n'interdit pourtant nullement le développement d'activités susceptibles de "susciter des émotions fortes et agréables ${ }^{10}$. La civilisation des mœurs, au contraire, ne peut être dissociée du développement de pratiques mimétiques qui « fournissent un cadre imaginaire destiné à provoquer une excitation imitant celle produite par des situations de vie réelle, tout en évitant ses dangers et ses risques $»^{11}$. Ces activités, parmi lesquelles on pourrait ranger le sport, la littérature (notamment le roman sentimental ou gothique), l'opéra, le cinéma, etc., ont « pour but de faire naître des émotions, de les éveiller, de provoquer des tensions sous la forme d'une excitation contrôlée et modérée - et ce sans les risques et les tensions habituellement liés à l'excitation qui accompagnent d'autres situations de la vie - , sous la forme d'une excitation 'mimétique' appréciable, celle-ci pouvant avoir un effet libérateur, cathartique, même si la résonance émotionnelle au cadre imaginaire contient, comme c'est en général le cas, des éléments d'anxiété ou de désespoir $»^{12}$. Autant dire que la civilisation des mœurs, à l'inverse d'une répression sans appel des émotions, encourage largement les individus à éprouver leur capacité à jouir de tensions excitantes produites selon des modalités bien maîtrisées. La sublimation et l'intellectualisation de la violence qui se joue à travers les pratiques mimétiques favorisent, en outre, une tendance croissante à l'introspection de soi et à l'exploration d'un " for intérieur » propre à l'individualisme moderne ${ }^{13}$. Norbert Élias a plus particulièrement analysé comment cette exigence

7 L'idée de cet article est née du constat des nombreuses homologies qui apparentent les critiques adressées à la corrida et à la chasse. En ce qui concerne ces dernières, je me permets de renvoyer à l'ouvrage que j'ai consacré à la genèse du mouvement Chasse Pêche Nature et Traditions, Les braconniers de la République. Les conflits autour des représentations de la Nature et la politique, Paris, Presses Universitaires de France, 2003.

8 Élias (N.), La solitude des mourants, Christian Bourgeois Editeur, 1987.

9 Ariès (P.), L'homme devant la mort. 2. La mort ensauvagée, Éditions du Seuil, 1977, p. 277.

10 Élias (N.), Sport et civilisation. La violence maîtrisée, Paris, Arthème Fayard, 1994, p. 119.

11 Ibid., 1994, p. 54.

12 Ibid., p. 62.

13 L'interdépendance qui lie systématiquement les analyses proposées par Norbert Élias commande que ses considérations sur la civilisation des mœurs ne soient pas dissociées de ses descriptions des dispositions propres à l'homo clausus, cette conception d'un moi séparé, autonome, original, extérieur et antérieur à l'ordre social. A ce propos, voir La société des individus, Paris, Fayard, 1991. 
d'une libération contrôlée des émotions individuelles est à l'origine du développement du sport moderne. Ce dernier, en effet, se caractérise par la mise en scène de combats imaginaires subordonnés à de nombreuses règles visant à garantir la beauté et l'attrait du jeu. L'intensité du plaisir final, en effet, dépend directement de la tension, de l'excitation suscitée, par le caractère fortement contraint et souvent imprévisible de la rencontre. La loyauté à l'égard des règles conditionne dès lors la possibilité de jouir aussi bien de la pratique que de la contemplation du spectacle sportif. Conformément aux valeurs de la civilisation, les activités sportives excluent les violences qui visent à porter atteinte à l'intégrité physique de l'adversaire mais célèbrent, en revanche, les luttes rudes mais euphémisées. Ce que l'on appelle aujourd'hui «l'esprit du sport» relève donc bel et bien de cette évolution générale des formes d'économies affectives édifiées, principalement à l'origine, par des élites aristocratiques de moins en moins guerrières. Sous l'effet de la civilisation des mœurs, les affects résultant des rapports agonistiques à autrui - l'agressivité, la rivalité, le défi, l'esprit de revanche — ont été transposés dans une sphère mimétique les dotant d'une tonalité différente qui autorise qu'ils soient mélangés à une sorte de délectation ${ }^{14}$. Cette jouissance sportive apparaît à l'analyse largement conditionnée par le respect de règles très précises : catégories en fonction de l'âge ou du poids, dimension strictement identique des équipes, gestes autorisés ou proscrits, alternance des positions sur le terrain afin que les éléments météorologiques ne puissent favoriser qui que ce soit, etc... D'une manière générale, ces dispositions réglementaires s'appliquent à strictement encadrer la possibilité de jouir d'une victoire sportive qui, en aucun cas, ne doit apparaître trop aisée ou escomptée. En matière de sport, la chasse vaut mieux que la prise, et seul le respect des conventions - qui interdisent toute victoire précipitée et inéluctable - peut garantir la tension dont dépendra l'intensité du plaisir final de la victoire. Autant dire que l'individu civilisé recherche dans les activités mimétiques non " pas le relâchement de la tension, mais au contraire, un type spécifique de tension, une forme d'excitation, souvent liée, comme Saint Augutin l'a si bien vu, à la peur, à la tristesse et à d'autres émotions que nous essayons d'éviter dans la vie ordinaire $»^{15}$. Les émotions qui, d'ordinaire, sont bridées ou censurées peuvent dès lors se manifester sans contrevenir aux préceptes de la civilisation : " les compétitions sportives permettent aux gens de remporter des victoires sur les autres lors d'un affrontement physique sans que les vainqueurs ne blessent physiquement les vaincus. La résolution de la tension de l'affrontement et l'effort à travers la victoire peuvent avoir un effet excitant et purifiant. On peut jouir de la confirmation de sa propre valeur sans mauvaise conscience, on peut goûter l'accroissement justifié de l'amour de soi dans la certitude que le combat a été loyal. De cette façon, le sport pourvoit à l'amour de soi sans mauvaise conscience ${ }^{16}$. Gardons nous pourtant de réduire l'émergence de pratiques mimétiques aussi importantes que le sport à des mécanismes exclusivement psychologiques. Le développement de la possibilité de jouir du spectacle sportif ne peut en aucun cas être dissocié, non seulement des contextes favorables au monopole de la violence par des structures politiques telles celles du parlementarisme, mais encore de la codification croissante de règlements propres à chacun des jeux sportifs : rugby, football, tennis, boxe, etc.... A ces règles écrites et uniformes, il convient d'ajouter encore l'institutionnalisation des éléments nécessaires à l'autonomisation du jeu: stades, championnats, corps arbitral, apprentissages spécifiques, etc... Le sport moderne se caractérise ainsi - et à l'encontre de nombreux jeux traditionnels - par des espaces et des temps clairement délimités et, par là même, distincts des rites festifs ou religieux.

\section{Maîtriser la violence du taureau, ou la civilisation des aficionados}

Les études consacrées à l'histoire de la tauromachie dans la péninsule Ibérique mettent en exergue de nombreux éléments qui semblent pouvoir être rapportés au tableau idéal-typique de la civilisation des mœurs ${ }^{17}$. Jusqu'à la fin du XVII ${ }^{\mathrm{e}}$ siècle, l'Espagne connaît de nombreuses formes

14 Élias (N.), Sport et civilisation, op. cit., p. 107.

15 Ibid., p. 110.

16 Élias (N.), op. cit., p. 64.

17 L'analyse qui suit, bien qu'elle n'engage que son auteur, est très largement redevable aux travaux de Frédéric 
populaires de jeux et rites taurins - taureau à la corde, courses des bêtes au cœur des villages, etc. - qui paraissent aussi peu codifiés et anarchiquement violentes que la Soule à laquelle Élias se réfère afin de souligner a contrario les caractéristiques du rugby moderne : aucune délimitation des espaces consacrés à la pratique, peu d'autonomie du jeu par rapport aux fonctions rituelles et aux finalités festives, indistinction des officiants et des spectateurs, diversités des usages locaux et absence de règles écrites. Les pratiques réservées à la noblesse espagnole, pour leur part, s'inscrivaient dans le prolongement de ces tournois chevaleresques au cours desquels s'exprimait « la magnificence des aristocrates qui démontraient devant le peuple leur valeur guerrière en terrassant des 'bêtes féroces' $\gg{ }^{18}$. A l'instar des pratiques liées à la chasse de l'époque, ces joutes étaient en grande partie perçues comme une forme de préparation à la guerre et d'exaltation des qualités martiales propres à la noblesse : jonchés sur leur monture, les chevaliers espagnols se devaient de transpercer des taureaux d'un coup de lance brutal et hardi. Les heurts violents de ces jeux taurins vont pourtant servir de support à la formalisation de la tauromachie à travers une série d'évolutions plus ou moins contingentes. Ainsi, Philippe V, petit fils de Louis XIV et premier roi Bourbon d'Espagne, n'appréciait guère les joutes violentes de la noblesse ibérique et interdit bien vite aux membres de sa cour de s'y adonner. L'importance sociopolitique des premières corridas l'incita pourtant à autoriser la noblesse espagnole à organiser des spectacles où officieraient exclusivement des roturiers d'origine populaire ${ }^{19}$. Les personnels des abattoirs fournirent ainsi un fort contingent d'officiants qui, sous le parrainage de la Maestranza de Caballerría sévillane ${ }^{20}$, furent chargés d'exalter les valeurs d'une aristocratie civilisée et civilisatrice. Cette dernière est ainsi à l'origine, non seulement de la construction des premières arènes spécialisées, mais encore de l'élevage prestigieux de longues lignées de taureaux dit de caste.

Le spectacle évolua ainsi très progressivement vers une forme de plus en plus codifiée inscrite - à l'instar des autres sports modernes - dans un temps et un espace autonome. Habits des protagonistes, enchaînement des séquences, rôles et obligations des officiants : l'ensemble de la gestuelle au sein de l'arène va faire l'objet d'une sophistication croissante. A l'orée du XIX ${ }^{\mathrm{e}}$ siècle, la première Tauromaquia (1796) n'est encore qu'une tentative isolée de rationaliser et de subordonner " les règles de l'art » de la corrida à des préceptes formels, explicites et esthétisants : définition des types de passes de cape, des coups de pique, de banderille ou d'épée ; typologie des divers comportements du taureau; division de la piste en trois zones distinctes (tablas, tercios, medios). Aujourd'hui, le déroulement de la corrida est strictement régenté par un Règlement taurin qui, en Espagne, relève d'une loi d'Etat contrôlée par le Ministère de l'Intérieur. Comme de nombreuses autres codifications sportives, ce règlement implique l'existence d'un corps de légistes chargé de veiller à leur respect. Ainsi, lors de leur entrée dans l'arène les matadors sont suivis d'alguaciles montés à qui revient la tâche de faire respecter les ordres de la présidence technique de la course. Bon nombre d'éléments prévus par le Règlement taurin se présentent sous les traits de conventions visant avant tout à brider le déploiement incontrôlé et inesthétique de la brutalité. A ce propos, les dispositions qui réglementent le premier «tiers », dit tercio des piques, apparaissent particulièrement significatives. Le Règlement taurin stipule, en effet, à quelle distance un picador (loyal à l'égard des règles) doit susciter la charge du taureau afin de s'exposer véritablement à la puissance de la bête. Il devra ensuite contenir sa charge aussi longtemps qu'elle se manifestera en s'interdisant de «vriller» sa lance dans le garrot du taureau ou de faire tourner son cheval afin d'amener le fauve à s'épuiser en poussant obstinément son adversaire. A la suite de «l'épreuve de la pique, le sang de l'animal, coule sur sa robe mais, dans l'idéal, le moins possible, les hémorragies trop voyantes, attribuées à l'ignominie du mauvais picador, étant vilipendées par les spectateurs ${ }^{21}$. L'objectif du picador, en effet, se veut essentiellement technique et vise par un cisaillement du

Saumade cités ci-dessous.

18 Saumade (F.), Les tauromachies européennes. La forme et l'histoire, une approche anthropologique, Paris, CTHS, 1998, p. 131.

19 Fournier (D.) et Saumade (F.), « L’artiste, le boucher et le sacrificateur », Etudes rurales, n¹13-114, 1989, p. 215.

20 Romero de Solís, «L'invention du 'ruedo'. La plaza de toros de Séville et les ruines de Pompéi », Gradhiva, 16, 1994.

21 Saumade (F.), Les tauromachies européennes, op. cit., p. 30. 
ligament nugal à interdire à la bête de porter la tête haute. Cet objectif, comme bien d'autres détails du spectacle, est subordonné à la volonté de mettre en scène la soumission progressive de la « sauvagerie » animale par la geste hautement maîtrisée de l'homme.

Cette "sauvagerie » attribuée à l'animal mérite plus particulièrement notre attention. Le taureau de combat, en effet, n'est en rien le représentant d'une espèce naturelle qui préexisterait au travail des hommes. Il est, au contraire, le produit d'une longue succession de sélections génétiques orchestrée par des éleveurs soucieux de « répondre aux impératifs techniques de la corrida espagnole ${ }^{22}$. La race du toro bravo a été ainsi forgée par plusieurs décennies de sélection des bêtes les mieux à même de transmettre à leur descendance les qualités requises par le spectacle tauromachique : mobilité, combativité, propension à baisser la tête. Ce savoir-faire déployé, dès le XVIII siècle, par les éleveurs andalous visa essentiellement à débarrasser l'espèce d'un tempérament " féroce " (fierezza) trop lié à cette violence imprévisible et donc incontrôlable que la civilisation des mœurs déprécie et honnit. La race modelée par l'ingénierie de l'homme se devait au contraire d'incarner cette bravoura qui, selon les aficionados, distingue les taureaux à la hauteur des exigences de la corrida artistique : combativité disciplinée, engagement franc et sincère, courage et résolution, absence d'une trop grande familiarité et connaissance de l'homme ${ }^{23}$. Bref, le taureau façonné par les éleveurs ne doit se confondre, ni avec un animal domestique trop apprivoisé, ni avec une bête dotée d'une férocité aveugle et désordonnée. Autant dire que l'ensauvagement de ce bétail domestique qu'est le toro bravo s'évertue avant tout à produire un animal capable de manifester les qualités les mieux adaptées à la mise en scène d'une violence maîtrisée par l'homme. En bas de soie rose et escarpin, le matador peut ainsi voir déferler sur lui une demi tonne de muscle symbolisant tout ce contre quoi l'homme civilisé se doit de l'emporter. Un ouvrage de vulgarisation, que l'un des "grands spécialistes de la tauromachie" destine à des publics néophytes, témoigne remarquablement du rôle que les « règles de l'art » de la corrida assignent au taureau.

La stratégie est simple à définir sinon à mettre en œuvre : asservir la charge, utiliser l'élan du taureau (...). Face au fauve, l'homme met en œuvre deux dominations. Sur lui même d'abord, en adoptant la tactique inverse à son réflexe immédiat, et qui seule garantit sa sécurité : l'immobilité statique de la sérénité et non la gesticulation et la fuite. Sur la bête, ensuite, pour infléchir sa course et sa charge, assouplir et incurver cette dernière, corriger ses défauts. La corrida met en scène les réflexes instinctifs des deux antagonistes : pour le taureau, charger droit ; pour l'homme, prendre ses jambes à son cou. L'art taurin exige du torero la double maîtrise de ces instincts : canaliser et arrondir la charge de l'animal, abolir son propre réflexe de fuite et le sublimer en une sérénité marmoréenne. Telle est la loi d'efficacité du combat (...). Déjà pointe l'enjeu du toreo : calmer le fauve, canaliser sa charge, le dominer pour créer, à travers lui, de la beauté (d13).

Il est manifeste ici que l'esthétique dont se réclame l'aficionado exalte ces valeurs cardinales qui, selon Norbert Élias, caractérisent la civilisation des mœurs. En premier lieu, cette capacité à subjuguer et commander une "sauvagerie", ici incarnée par le taureau : "l'homme guide le fauve d'une simple et régulière flexion du poignet, au rythme de la rotation de son bras (...). L'homme fait le pari de détourner cette charge sur le drapelet, de maîtriser l'élan du taureau en conduisant ses pas » (d13). Par ailleurs, il s'agit également ici de célébrer la maîtrise de soi, l'aptitude à dompter les inclinations les plus immédiates, à ne rien laisser transparaître qui puisse relever d'un comportement trop instinctif. Lors de la préparation de ses passes de cape, l'homme se doit « de trouver le sitio, sa place par rapport au taureau, à l'exacte distance qui convient à la vue de la bête, à la longueur de charge, à son souffle. Mais aussi la place du torero par rapport à lui-même : bien dans sa peau, confiant, dominateur de soi pour l'être du fauve » (d13). Les vrais connaisseurs loueront plus particulièrement l'élégance et la sobriété du maestro, son absence de jeux de jambes nerveux, son refus d'insister "sur les exercices physiques et virevoltants ou sur les séquences à genoux qui veulent évoquer l'imminence du danger $»^{24}$. Le « grand toréador » se reconnaîtrait ainsi à son aptitude à instaurer « une sorte de langueur intemporelle, dès lors qu'intervient la notion de temple (du verbe templar : accorder, tempérer, adoucir)» (d13). Ce modèle idéal — certes, souvent

22 Fournier (D.), « Le toro bravo, sujet d'ethnographie », Gradhiva, 16, 1994, p. 46.

23 Saumade (F.), Des sauvages en Occident. Les cultures tauromachiques en Camargue et en Andalousie, Paris, MSH, 1994, p. 42 et s.

24 Saumade (F.), Les tauromachies européennes, op. cit., p. 37. 
démenti par les contingences du spectacle - est celui qui caractérise la forme canonique de la corrida andalouse. Frédéric Saumade a remarquablement montré tout ce que l'édification de cette dernière doit à un souci d'opposition et de distinction vis-à-vis des jeux taurins plus populaires, souvent perçus comme vulgaires, violents et de mauvais goût ${ }^{25}$. Par ailleurs, bon nombre d'indices attestent à quel point cette corrida dite artistique s'est progressivement dépouillée des éléments les plus brutaux et sanguinaires qui émaillaient autrefois ses mises en scène. Citons, par exemple, la disparition du coupe-jarret, long manche doté d'une lame en demi-lune permettant de sectionner les pattes des taureaux afin de mieux les faire plier devant l'homme ; ou bien encore le sort réservé aux bêtes jugées insuffisamment combatives, à savoir le lâcher de chiens ou la pose de banderilles enflammées. L'invention en 1921 du carapaçon visant à protéger les chevaux des picadors et dont l'usage est devenu obligatoire depuis 1928, témoigne de l'aversion croissante à l'égard du sang au regard même des aficionados les plus avertis. Auparavant, faut-il le rappeler, bon nombre de chevaux finissaient éventrés et devaient fuir les assauts du taureau en piétinant leurs propres entrailles. La disparition de telles scènes a sans doute facilité l'édification d'une esthétique tauromachique pour qui la mort de l'animal ne constitue qu'un élément secondaire entièrement subordonné à une émotion décrite comme grave, intense, et sans pareil.

A certains moments de la corrida, tout s'arrête une fraction de secondes. Souffle coupé. Le mouvement de l'homme et de la bête se fige sur votre rétine. Un frison vous parcourt. Cet état, intense et bref, comme le plaisir, précaire et indéfinissable comme lui, voilà ce qui crée et alimente l'afición (d13).

Bien d'autres témoignages de ce type décrivent la phénoménologie du spectacle tauromachique selon des modalités qui semblent bel et bien attester de la "correspondance complexe entre la dynamique de l'évènement mimétique lui-même et la dynamique psychologique du spectateur $»^{26}$. L'étiquette exigeante et les règlements qui régulent le toréo nourrissent cette tension, cette excitation agréable, qui constitue selon Norbert Élias le ressort essentiel des affrontements sportifs corporels codifiés et réglés. Lors d'une corrida, en effet, trois matadors s'affrontent, trois hommes se plient aux règles de l'art tauromachique afin de tenter de se dépasser et de se surpasser en habileté. Comme dans tout autre spectacle sportif, la satisfaction des spectateurs sera directement indexée sur la capacité des protagonistes à se plier à ces règles dont dépend l'évaluation de leur maestria. Pour résumer, les valeurs sous-jacentes à la corrida peuvent sans aucun doute être envisagées comme le produit d'une forme particulière — nationale et (inter)régionale — de ce processus de civilisation que Norbert Élias a toujours perçu dans sa diversité. Ce point méritait d'autant plus d'être relevé que, comme on le verra, les critiques adressées à la tauromachie apparaissent, elles aussi, largement dépendantes d'une forme d'interprétation des préceptes prescrits par la civilisation des moeurs.

\section{Dénoncer les « barbares », ou la civilisation de l'animal protégé}

Les dénonciations de la tauromachie nous offrent incontestablement un cas exemplaire d'une modalité de construction des causes militantes aujourd'hui très courante (notamment dans le domaine de la protection animale) : la sensibilisation du public. Il faut entendre par là, plus précisément, que les entrepreneurs de cause s'efforcent de mobiliser leurs contemporains en faisant appel à des « sensibilités communes sur lesquelles peuvent s'appuyer des accords préréflexifs $3 / 4$ de l'ordre, si l'on veut du préjugé, pour ne pas dire du préjugement $3 / 4$ entre des personnes qui se reconnaissent, sinon les mêmes valeurs éthiques, au moins une communauté de réaction, que l'on appelle souvent "viscérales" pour dire qu'elles préexistent en sorte à leur justification par des principes $»^{27}$. Le tableau idéaltypique emprunté à Élias nous permettra d'examiner dans quelle mesure ces sensibilités communes semblent bel et bien résulter d'une forme particulière de

25 Saumade (F.), Les tauromachies européennes, op. cit., p. 178 et s. L'auteur souligne également comment — dans leur opposition à la «tauromachie esthétisante et idéalement douce » andalouse - les courses portugaises, aragonavarraises, landaises et camarguaises ont eu tendance, en revanche, à (re)valoriser des formes de violence spectaculaires et métaphoriques.

26 Élias (N.), Sport et civilisation. La violence maîtrisée, op. cit., p. 114.

27 Boltanski (L.), La souffrance à distance. Morale humanitaire, médias et politiques, Éditions Métailié, 1993 , p.85. 
déclinaison des valeurs de la civilisation des mœurs. La lecture des critiques adressées à la tauromachie, en effet, a vite fait de révéler à quel point la réprobation du sort réservé au taureau ne constitue que l'un des éléments d'un système complexe de prescriptions morales interdépendantes : la dépréciation de l'agressivité, l'occultation du sang et de la mort, l'exigence d'un contrôle constant des «pulsions » immédiates, l'encadrement des affects agonistiques par des conventions communément admises. Bien que ces éléments apparaissent souvent inextricablement liés, nous les examinerons consécutivement afin d'expliciter notamment le rôle que l'économie émotionnelle sous-jacente à la dénonciation de la corrida assigne au toro bravo. Pour être plus précis, nous compléterons ce système de valeurs par une hypothèse indispensable à une bonne compréhension de l'aversion souvent suscitée par la corrida : les économies affectives modelées au fil du temps par la civilisation des mœurs doivent sans doute être rapprochées du développement de cette politique de la pitié qu'Hannah Arendt observe, plus particulièrement, depuis le milieu du XIII ${ }^{\mathrm{e}}$ siècle $^{28}$. En d'autres termes, les façons d'être concerné et affecté par le spectacle de la souffrance d'autrui ont sans aucun doute été façonnées par la dépréciation de la violence, par l'intensification de la pudeur, ou bien encore par l'exaltation aussi bien du tact à l'égard d'autrui que de l'introspection de soi. De fait, et comme on le verra, les critiques de la tauromachie empruntent énormément à l'appel à la compassion et à la célébration d'un homme sensible et civilisé qui se doit de s'émouvoir du sort des malheureux.

Les documents militants des opposants à la tauromachie recèlent souvent une iconographie mettant très largement en exergue le caractère sanglant, pour ne pas dire, sanguinolent, de la pratique. Le logo du Front de liaison anti-corrida, par exemple, présente un taureau couvert de banderilles qui baigne pratiquement dans son sang. Sur le site de cette fédération d'associations, les auteurs du texte «La corrida négation des droits de l'animal» illustrent leur texte, non seulement de photographies de taureaux profondément blessés, mais encore d'animations simulant une plaie d'où s'échappe un flux continue d'hémoglobine. Il est évident que ces éléments en appellent avant tout au dégoût du sang que les valeurs de la civilisation exigent habituellement de chacun. D'ailleurs, si dans son premier ouvrage, La mort donnée en spectacle, Claire Starozinski utilise une iconographie du même type, le bon de commande de son deuxième pamphlet anti-corrida précise soigneusement que «l'ouvrage ne contient aucune photo choquante, vous pouvez donc le commander sans crainte de voir votre sensibilité mise à rude épreuve ». Comme on peut s'en douter, autant que le sang, la souffrance et la mort, font l'objet de nombreuses descriptions visant à débusquer les excès malséants de la corrida. Cette dernière est ainsi systématiquement présentée comme une « torture donnée en spectacle » (d2) :

Enfin un livre radical et argumenté, qui rabaisse la corrida, "érigée en art et en culture", à ce qu'elle est vraiment : une torture ! (d4)

La corrida, ce rite sanglant que $83 \%$ des Français réprouvent, consiste à torturer six taureaux durant un quart d'heure chacun (d5).

Une corrida de toros (dite aussi corrida formelle) c'est le supplice de six taureaux torturés, jusqu'à ce que mort s'ensuive, par trois équipes de toreros (d6).

Elle est cette pratique tribale qui consiste en l'abattage rituel de six taureaux à chaque exhibition (payante), abattage assorti de tortures préalables (d9).

La féria de Nîmes — fête de la douleur et de la mort — durant laquelle ont été torturés jusqu'à quatre vingt dix taureaux en quelques jours (d1).

A la fin du deuxième tercio, le supplicié est hérissé de banderilles et épuisé tant par les courses-poursuites que par ses blessures et le sang perdu (d7).

Cette omniprésence de l'image de la torture - qui sous-entend la présence d'un bourreau et d'une victime - mérite d'être commentée car elle mobilise deux émotions au centre des processus de la civilisation des mœurs. En tout premier lieu, la répulsion à l'égard de la souffrance occasionnée par une agression portant atteinte à l'intégrité physique des êtres. En second lieu, la pitié pour le malheureux exposé à une brutalité gratuite. Ainsi, de nombreuses descriptions s'appliquent avant tout à présenter les taureaux sous les traits de victimes d'autant plus innocentes que rien, en « vérité », ne les prédisposent à ce traitement révoltant.

28 Boltanski (L.), La souffrance à distance, op. cit., p. 15 et $\mathrm{s}$. 
Le taureau est un paisible herbivore qui n'attaque jamais l'homme mais fuit devant lui (d9).

Le taureau dit de corrida n'est pas un bovin à part avec un "génome de l'agressivité". (...) Le taureau n'est pas un fauve et encore moins une bête féroce. Domestiqué par l'homme, il n'est donc pas un animal sauvage. Il est d'un naturel paisible et aspire à la tranquillité. Comme les autres herbivores, face à un danger, il privilégiera la fuite (d10). paix (d3).

[Pour Théodore Monod, il convient] d'hâter le jour où ces bovidés domestiques pourront enfin brouter en

La corrida consiste à soustraire un être vivant sensible (en l'occurrence le taureau) à son environnement naturel pour le placer dans des conditions artificielles où il ne pourra rien faire d'autre que de subir le bon vouloir de ses tortionnaires (d9).

Il est évident ici que cette manière de présenter le taureau s'efforce de faire participer ce que l'on appelle habituellement l'opinion publique à un «mouvement émotionnel » qui, dans le même temps, justifie le caractère impérieux de l'activisme des anti-corrida. A travers de tels discours, en effet, la pitié pour les taureaux " auxquels on peut faire subir les pires sévices » (d7) est invitée à se muer en une colère qui invite à l'action collective au côté des promoteurs de la cause défendue. Pour ce faire, l'expression très ostensible de l'indignation doit préalablement favoriser « une réorientation de l'attention, qui se détourne de la considération déprimante du malheureux et de ses souffrances pour aller chercher un persécuteur et se centrer sur lui ${ }^{29}$. C'est pourquoi bon nombre des pamphlets contre la corrida se présentent-ils sous les traits d'une accusation méthodique de tous ceux qui la supportent (au double sens du terme). Au cours de ce procès, les « acharnés de la torture donnée en spectacle » (d11) apparaissent bel et bien jugés à l'aune des impératifs moraux que les opposants à la tauromachie déduisent des valeurs de la civilisation des mœurs. Ainsi, et en tout premier lieu, les adeptes de la corrida - matadors comme spectateurs - sont systématiquement dénoncés en tant que vecteurs d'une violence qui, loin de s'exercer sur les seuls taureaux, menacerait l'ordre social dans son ensemble. Pour les membres de la FLAC, par exemple, le simple fait de présenter «la tauromachie comme un spectacle admirable correspond à une forme très pernicieuse de banalisation de la violence » (d11) :

La place privilégiée qu'occupe aujourd'hui la corrida dans les media tend à déculpabiliser celles et ceux qui vont y assister. Mais nous devons nous interroger sur la réelle demande en matière de violence dans nos sociétés (d9).

La corrida ne se contente pas de banaliser la violence, le sang, la torture, le meurtre : elle les glorifie, les élève au rang de la culture et des beaux-arts (d9).

Je suis viscéralement opposé à la corrida, spectacle abject qui réveille les pires instincts chez l'être humain : sadisme, violence, voyeurisme... (d11).

Cette dénonciation de la violence mérite deux remarques. Premièrement, l'excès de brutalité stigmatisé ici ne concerne pas seulement celle qui s'exerce sur l'animal mais presque tout autant celle qui heurte et corrompt la sensibilité du spectateur «normalement » constitué : «la réalité dépassant de loin l'imagination, je déconseille aujourd'hui à quiconque - écrit la pasionaria de l'anti-tauromachie - de pénétrer, ne serait-ce une fois, dans l'arène pour éviter d'être confronté à pareille violence » (d2). A la lumière de ce conseil, on ne s'étonnera pas d'apprendre que les statuts de l'Alliance pour la suppression des corridas prévoient de faire entrer dans son objet la protection « de tout [spectateur] de moins de seize ans contre une quelconque atteinte morale, psychologique (...) survenue ou susceptible de survenir au cours d'un spectacle tauromachique » (d4). Deuxièmement, la critique de la corrida consiste à dénier la dimension contrôlée et mimétique d'une violence spectaculaire soupçonnée de pouvoir contaminer le domaine des relations humaines ordinaires : «l'absence de compassion envers les animaux m'a toujours paru suspecte et je demeure persuadée qu'un individu qui cautionne les sévices infligés aux animaux cautionnera, de la même manière, les sévices infligés aux humains, si un jour la conjoncture s'y prête » (d2). Autant dire qu'il est ici bel et bien question d'apprendre aux hommes à maîtriser une violence qui - en l'absence d'un autocontrôle exigeant - menace sans cesse de ressurgir de manière inquiétante. A ce propos, le diagnostique de la "Commission éthique» de la FLAC est sans appel : « le sadisme, dans une corrida, ne vise pas seulement les bêtes : les spectateurs exigent des taureaux redoutables, chahutent

29 Boltanski (L.), La souffrance à distance, op. cit., p. 91. 
les toreros prudents, révélant ainsi leur désir inavoué de voir des hommes étripés. La nature humaine comporte une agressivité qui, si elle n'est pas éduquée, maîtrisée, peut dégénérer en sadisme » (d9). Les militants de l'Alliance pour la suppression des corridas, de leur côté, n'hésitent pas à surenchérir en démasquant les véritables motifs qui pousseraient les organisateurs à faire toréer des enfants : " le public a besoin de sensations de plus en plus fortes. L'arène n'est-elle pas l'ultime endroit où l'on peut encore, en toute légalité, se délecter de la mort de l'animal et parfois en prime de celle d'un homme ? Alors, un enfant, pensez donc !» (d5). Bien d'autres éléments de ce type trahissent à quel point les réquisitoires contre la tauromachie entendent avant tout dénoncer une nature humaine imparfaitement civilisée qui « suggère de très sombres réflexions sur le tréfonds du "coeur humain" » (d7).

Les spectateurs qui applaudissaient autrefois les massacres des gladiateurs sont les mêmes qui jouissent, aujourd'hui, de la mise à mort du taureau. Le goût du cadavre, de la souffrance et du sang n'a pas vieilli. Simplement, derrière les ors, la lumière, la fanfare et de fallacieux prétextes, s'exprime une passion morbide (d1).

Ce spectacle, dont les écrans dispensent des bribes, occultant la cruauté et la violence la plupart du temps, intrigue assurément. Il revêt cet aspect quelque peu sulfureux dont raffolent les sociétés prétendument aseptisées. Il fait appel à ce fonds résiduel de perversion et d'ambiguïté qui sous-tend l'individu (d9).

La divinisation de la tauromachie n'est qu'un paravent qui sert à dissimuler des pulsions primaires et une esthétique de mauvais aloi. Je ne doute pas que le simple bon sens ne finisse par prévaloir contre ces raisonnements spécieux (d2).

On aura sans doute remarqué ici que l'invocation de la «nature humaine » et des « instincts » prétendument primordiaux ne vise aucunement à concéder des circonstances atténuantes aux adeptes de la corrida. Bien au contraire, il s'agit avant tout - en conformité avec les valeurs de la civilisation - de les condamner sous plusieurs motifs d'accusation étroitement complémentaires. En tout premier lieu, les aficionados sont incriminés en raison de leur incapacité à faire preuve de retenu et à maîtriser des désirs trop immédiats. De là, résultent les intonations puritaines des condamnations sans appel des plaisirs suspects des « tauromaniaques » (d7); des condamnations qui peuvent d'ailleurs aussi bien se fonder sur le magistère de l'église que sur l'autorité de sciences « hygiénistes ».

Les aficionados sont une minorité, mais une minorité agissante organisée et motivée par le plaisir (d2).

Car sexe et fétichisme sont les deux mamelles de la corrida (...). Ce monde éminemment machiste abrite en son sein de délicates perversions (d1).

[Selon Théodore Monod], il s'agit de permettre à une catégorie très limitée de satisfaire son appétit de sang et de cruauté [il convient donc de s'insurger] contre ce qu'un clairvoyant évêque de Nîmes avait osé dénoncer comme une cruelle et scandaleuse complicité entre sadisme et érotisme (d1).

[Dans Le Petit manuel à l'usage des anti-corrida, l'auteur cite la lettre pastorale écrite, en 1863, par l'évêque de Nîmes] «Ce sont surtout les inquiétudes ou les douleurs des taureaux qui vous passionnent... Et l'on oserait dire après cela que des chrétiens peuvent décemment assister à de pareilles scènes ? Du côté du spectacle, rien n'est digne du chrétien parce que tout est frivole et barbare... Tel est l'incendie allumé par tous les spectacles sanglants. Quand on les a vus on veut les revoir encore... le sang, une fois bu par les yeux, excite en eux une soif intarissable. Plus on la satisfait, plus elle est exigeante, plus aussi elle rend impitoyable » (d1).

La psychiatrie de la corrida [écrit un certain Professeur Heuse] relève du sadisme, où l'on distingue l'homosadisme pratiqué sur un être appartenant à la même espèce, et l'hétérosadisme pratiqué sur un être appartenant une autre espèce (...). Dans le cas de la corrida, il s'agit de sadiques visuels qui assistent à des tortures infligées par l'homme à l'animal (cité en d1).

L'enfant [ajoute le professeur et neuropsychiatre Didier-Jacques Duché] est un pervers polymorphe - qui accomplit par plaisir et sous diverses formes des actes cruels. Comment dès lors accepter, voire même favoriser tout ce qui exalte la violence, la lutte sanguinaire ? Un tel spectacle est d'autant plus nocif qu'il entraîne des manifestations d'hystérie collective auxquelles le jeune enfant est particulièrement sensible. Aussi me semble-t-il absolument nécessaire d'étendre à la tauromachie la censure concernant les enfants (cité en d1).

Les enfants qui y sont entraînés par leurs parents se voient, de ce fait, inoculer une fascination certaine pour la violence (...). Il est permis de se demander si l'étalage de la torture d'animaux ou même son évocation n'a pas, sur les jeunes esprits, au jugement encore vacillant, d'effet aussi pervers que celui de la pornographie (d2).

Dans une société où l'on essaie d'endiguer la violence, surtout chez les jeunes, les adultes que nous sommes portent une très lourde responsabilité en encourageant et en incitant de très jeunes enfants à faire subir des sévices à des animaux avant de les assassiner (...). Ces écoles sont donc celles du sadisme dans lequel bien souvent les enfants excellent spontanément. Les y encourager est donc doublement criminel. Et les pouvoirs publics qui subventionnent ou laissent faire dans le meilleur des cas, cautionnent ce qui non seulement est condamnable mais 
relèvent carrément de la psychiatrie (d8).

Il serait sans doute fastidieux de relever ici d'autres, et très nombreux, éléments qui témoignent du caractère alarmant que les anti-corrida attribuent à la fascination que la tauromachie est susceptible d'exercer sur des « esprits jeunes et vulnérables, encore incapables de discernement»(d9). Ce thème omniprésent à d'ailleurs donné lieu, au sein de la FLAC, à la création d'une « commission éducation. Réseau de vigilance pédagogique » animée par un enseignant du secondaire qui exhorte ses collègues de la France entière à s'insurger contre le "prosélytisme tauromachique en milieu scolaire »(d11). Contentons-nous de noter que ces préoccupations pédagogiques corroborent remarquablement les analyses de Norbert Élias selon lesquelles l'accentuation des sentiments de pudeur et d'embarras face aux conduites dépréciées par la civilisation implique une occultation plus grande encore dans le cadre de l'éducation des enfants. Le sociologue allemand souligne d'ailleurs, à ce propos, que «si la dissimulation et le secret qui entouraient jadis le domaine sexuel se sont heureusement relâchés, les tabous sociaux se sont déplacés et singulièrement renforcés en ce qui concerne la mort. L'attitude pusillanime de refus et de gêne qui entoure aujourd'hui la fin d'un être humain [mais aussi de l'animal, dans le cas qui nous intéresse ici] est tout à fait comparable à celle qui prévalait dans le domaine sexuel à l'époque victorienne $»^{30}$. D'une manière générale, les valeurs de la civilisation ont toujours exigé que les « bonnes manières » soient inculquées aux individus dès leur plus jeune âge afin qu'ils ne puissent risquer d'en ignorer les bienfaits tout au long de leur vie. Dans le cas qui nous intéresse, le spectre d'une éducation défaillante est effectivement très souvent évoqué afin de fustiger, non seulement une appétence déplorable pour la violence et le sang, un individu livré à des pulsions les plus primaires, mais encore une piètre aptitude à cette introspection qui caractériserait l'homme véritablement maître de soi.

Les écoles taurines ou l'initiation au sadisme (...). Dès l'âge de deux ans, certains ont déjà vu leur première corrida, alors que nos sociétés sont plus sévères pour les autres formes de violence susceptibles d'être vues par des adolescents ! L'enfant est charmé par l'ambiance, la musique, les costumes. Il est impressionné par ce taureau noir qui déboule du toril, entrant dans l'arène. Les adultes lui parlent en termes élogieux de la corrida en occultant totalement la souffrance du taureau (...). Il commencera sa morbide formation en s'entraînant sur une botte de paille (...). Ensuite, lorsqu'il aura acquis assez d'expérience, l'étudiant en atrocité devenu "novillero" participera à des "novilladas" sans picador avec de jeunes taureaux ou "novillos" âgés de deux à trois ans ; puis des "novilladas" piquées avec des "novillos" de moins de quatre ans (...). Avec les "novilladas" commence le rituel de la mutilation de la victime ; un procédé traditionnel chez les "serial-killer". L'élève "méritant" a droit à ses premiers macabres trophées : une ou deux oreilles, ou la queue, ou les trois à la fois ! (d8).

Le torero prend plaisir à se faire peur, à être admiré, à dominer et enfin à vaincre la bête. Il est absolument persuadé d'aimer cet animal au-delà de tout car seul ce dernier lui permet de réaliser " son côté sombre » et d'assouvir des pulsions dont il n'a, le plus souvent, aucune conscience (d2).

Nous dirons des motivations des spectateurs adultes et adolescents qu'elles sont de l'ordre de l'inavouable, mais soigneusement occultées par les inconditionnels qui ne souhaitent pas davantage faire à ce sujet un travail d'introspection (d9).

Il faut n'avoir jamais réfléchi au mystère de la vie et de la mort pour ravaler celle-ci au rang de spectacle assouvissant les pires pulsions (d3).

Par voie de presse, j'ai conseillé aux donneurs de leçons de se titiller un peu la conscience, si toutefois ils en ont une... (d1).

On peut également remarquer comment les critiques de la tauromachie tendent à édifier une conception de la « civilisation » qui porte les opposants à la corrida à s'attribuer une impérieuse et urgente mission. A la différence de la théorie de Norbert Élias, cette conception des plus prescriptives érige la civilisation en un mouvement univoque, inéluctable, que les " hommes de bien » se doivent de propager auprès de leurs contemporains les plus attardés. Les missionnaires de la cause animale se font ainsi un devoir de ravaler la passion pour la corrida au rang des scories de l'histoire dont il conviendrait d'hâter « l'abolition » (d6).

Tout individu qui pratique la torture ou qui se délecte de son spectacle fait obstacle au progrès de la civilisation. Un progrès qui implique, partout et en toutes circonstances, l'aide à la souffrance, y compris celle de l'animal (d2).

Aujourd'hui, je me sens à la fois affligée et confiante. Affligée de constater que certains de mes contemporains peuvent encore se délecter de spectacles barbares alors que tant d'autres possibilités de

30 Élias (N.), La solitude des mourants, Christian Bourgeois Éditeur, 1987, quatrième de couverture. 
divertissement s'offrent à eux (...). Mais confiante parce que notre combat va dans le sens de l'inéluctable évolution des mœurs et je ne vois pas comment la corrida pourrait avoir le moindre avenir ... [Les cotisations des sympathisants pourront permettre] tous ensemble d'éradiquer la corrida, véritable fléau indigne de notre civilisation (d2).

Votre combat, Claire Starozinski, se place à la pointe de l'évolution de nos mœurs et de la psychologie qui n'admettront plus la survivance à des fins mercantiles, de rites sanglants dans l'accomplissement desquels se perpétue l'Antiquité païenne. Angelo Rinaldi (d3).

La société [affirme Nicolas Hulot dans un message de soutien aux anti-corrida] grandira à se débarrasser de comportements décadents (d1).

[Une tradition] cesse d'être respectable et doit être combattue dès lors qu'elle ne correspond plus à l'évolution des mœurs résultant des progrès de la civilisation (...). Je redoute que le culte de la tauromachie n'enfonce Nîmes dans une image de ville passéiste étrangère à l'évolution du monde (...). Que quelques nostalgiques d'une époque ou d'un courant entretiennent le culte du passé ne saurait être assimilé à l'existence d'une tradition vivante $(\mathrm{d} 2)$.

Si l'on veut à toute force considérer la tauromachie comme un art, il faut alors admettre que ce ne peut être qu'un art décadent, représentatif de la morbidité dont a souffert l'Espagne aux heures les plus terribles de son histoire et qui transparaît dans certaines toiles de Vélasquez ou de Goya (d2).

Au temps bibliques, chez les Israélites, ce fut la tradition du bouc émissaire, que l'on condamnait après l'avoir chargé de tous les péchés. C'est encore, de nos jours, chez les musulmans le prétexte pour égorger des milliers de moutons à l'occasion de l'Aïd-el-Kébir (d2).

En présence de tels propos, il peut être tentant de conclure, à la suite de Paul Yonnet, que « sous le pan-universalisme abstrait de la zoophilie contemporaine avancent des pratiques de 'protection animale' totalement sectaire à l'égard de la variété culturelle des sociétés humaines, en bref, on assiste par cette voie au retour des pratiques jacobino-coloniales du XIX ${ }^{\mathrm{e}}$ siècle, à la désintégration des inhibitions sociales par quoi avait pu prévaloir au nom du respect des cultures et des ethnies, l'idée d'une nécessaire différenciation des traditions populaires $»^{31}$. Pourtant, cette manière d'en appeler à la suprématie éthique du pluralisme culturel équivaudrait sans doute à renoncer à la neutralité axiologique à laquelle cet article voudrait à tout prix s'en tenir. Le dit article, faut-il le rappeler, se propose - non pas d'arbitrer les conflits de valeurs étudiés en débusquant les travers d'un ethnocentrisme intolérant - mais bien plutôt d'examiner le statut que diverses économies affectives «civilisées » attribuent à l'animal. De ce strict point de vue, les analyses précédentes peuvent être aisément résumées :

- pour les aficionados, le taureau symbolise une sauvagerie animale que l'homme se doit de défier et de soumettre tout en se pliant à des conventions qui visent à magnifier l'autodiscipline, la finesse et la virtuosité de sa maîtrise.

- pour les opposants à la tauromachie, le taureau incarne la victime en proie à une sauvagerie humaine inquiétante et qui, compte tenu de son caractère incontrôlée et incontrôlable, se doit d'être jugulée.

En d'autres termes, par delà leurs divergences, ces deux conceptions concurrentes résultent de valeurs communes liées aux processus complexes de la civilisation des mœurs. La différence notable entre les deux perspectives, en définitive, repose sur l'extension en direction du monde animal des préceptes de justice et de pitié que les opposants à la corrida se refusent de réserver à la seule humanité. Au regard de ces derniers, en effet, la pacification des attitudes, la sublimation et la retenue dans les émotions, l'introspection de soi conduisant au respect d'autrui, devraient s'appliquer indistinctement aux mondes des hommes comme des animaux.

Le monde humain et le monde animal occupant l'espace Terre doivent pouvoir y cohabiter pacifiquement

Théodore Monod parla d'hominisation et de réconciliation des divers mondes du vivant au cours de ce qui devait être l'une de ses dernières interventions publiques (d12).

Je fais partie de ceux qui ressentent de l'empathie pour tout ce qui vit, c'est-à-dire la capacité de se mettre à la place de l'Autre, contrairement aux persécuteurs qui, eux, ne se représentent jamais la souffrance de leur victime. Le fait que la violence et l'humiliation s'exercent sur la vie, qu'elle soit animale ou humaine, est intolérable et on se doit de combattre de tels comportements (d2).

31 Yonnet (P.), Jeux, modes et masses. 1945-1985, Paris, Gallimard, p. 227. 
Je me garderai [écrit Jacques Julliard] de toute comparaison indécente mais, hier, on s'indignait que les hommes fussent traités comme des bêtes. Pouvons-nous accepter que les animaux soient traités aujourd'hui comme jadis une partie de l'espèce humaine ? (d3)

Les opposants à la tauromachie semblent ainsi revendiquer que les hommes se conduisent à l'égard des animaux en respectant les conventions que ces derniers pourraient contribuer à définir si... seulement ils le pouvaient ! En l'absence d'une telle condition, comment ne pas être révolté à l'idée que la corrida puisse impliquer, malgré lui, un « être vivant muet» (d1) ? A ce propos, la critique de la corrida au nom des valeurs de la sportization s'avère des plus significative. Alors que - comme nous l'avons remarqué plus haut - la tauromachie relève de cette codification de combats mimétiques entre trois hommes rivalisant en dextérité, les pamphlets anti-corrida ne cessent de dénoncer un face à face entre l'homme et l'animal aucunement encadré par la reconnaissance préalable de règles communément acceptées.

Le boxeur ou le rugbyman se prononcent pour aller sur le ring ou sur le terrain de sport en toute connaissance de cause, personne n'a décidé pour lui, il prend donc ses responsabilités pleines et entières, avec un beau paquet d'argent à la clef, ne l'oublions pas. Le taureau, lui, ne gagnera que sa mort certaine, après un quart d'heure de souffrance (d1).

Les tauromaniaques prétendent bien hypocritement que la corrida est un combat loyal dans lequel l'homme et l'animal ont des chances égales. Il n'en est rien (...). Six hommes armés, longuement entraînés et agissant de concert contre une bête seule, novice et ignorante de tout, c'est ce que certains appellent un combat loyal, à armes égales (d7).

Comment [avec la pratique frauduleuse de l'afeitado consistant à scier l'extrémité des cornes] oser prétendre que la corrida est un combat à armes égales ? (...) On constate que de plus en plus de taureaux manquent de vitalité. Peut-on, dans ces conditions, parler de combat loyal ? (d2)

Les toreros, qui peuvent gagner jusqu'à 1600000 francs pour une seule prestation, prennent de moins en moins de risques en exigeant ces amputations ignobles, d'autant que de 1948 à 1993, on a enregistré une mort de torero pour 34.033 taureaux tués en France et en Espagne (...). Comment oser dès lors, parler d'un « combat d'égal à égal » (d5).

A l'encontre de «l'esprit du sport», la tauromachie serait ainsi coupable de ne pas permettre l'annulation des différences - en l'occurrence, entre l'homme et l'animal — au profit d'une égalité des chances seulement rompue par une confrontation loyale des habilités mises en jeu. Comment dès lors se satisfaire d'une tension mimétique créée par un affrontement dont l'issue — la mort d'un des protagonistes - n'est pas incertaine ? La véritable loyauté d'ailleurs n'exigerait-elle pas que les chances de succomber soient égalitairement réparties entre le taureaux et le matador qui « ne risque que très rarement sa vie » $(\mathrm{d} 1)$ ?

Le cadre théorique emprunté à Norbert Élias paraît donc particulièrement apte à mettre en exergue les fondements des controverses autour de la corrida. Un bon usage de la sociologie évolutionnelle devrait, en outre, nous interdire de surestimer le caractère nouveau des différends qui opposent les économies affectives des aficionados et des détracteurs de la tauromachie. Dès le début du XIX ${ }^{\mathrm{e}}$ siècle, en effet, ces deux systèmes d'attitudes semblent avoir entamé des évolutions parallèles qui ne leur ont jamais permis de se rejoindre ${ }^{32}$. La tauromachie, comme nous l'avons noté plus haut, n'a cessé de réduire les éléments les plus brutaux et sanglants de ses mises en scène spectaculaires. La compassion à l'égard de l'animal, de son côté, semble avoir été principalement marquée par un processus de vulgarisation et de diffusion qui lui a permis d'accéder au rang des valeurs communes les plus hégémoniques. Maurice Agulhon ${ }^{33}$ et Valentin Pelosse ${ }^{34}$, démontrent tous deux à quel point les préoccupations concernant les mauvais traitements envers les animaux ont pu se manifester tous

32 Soulignons bien que ce propos se distingue nettement de l'interprétation évolutionniste des anti-corrida selon laquelle leurs dispositions seraient plus récentes, mieux achevées, « moins archaïques », que celles des « amateurs de barbarie » (d4) du monde taurin.

33 Agulhon (M.), «Le sang des bêtes : le problème de la protection des animaux en France au XIX ${ }^{\mathrm{e}}$ siècle », dans Histoire vagabonde, tome I, Paris, Gallimard, 1988.

34 Pelosse (V.), «Imaginaire social et protection de l'animal. Des amis des bêtes de l'an X au législateur de 1850 », L'Homme, XXI (4), 1981. L'auteur analyse les propos recueillis dans 27 mémoires manuscrits présentés dans le cadre d'un concours de l'Institut en 1802. La question posée aux candidats porte sur les mauvais traitements envers les animaux et sur la nécessité, en la matière, de légiférer dans un souci de morale publique. 
au long du XIX ${ }^{\text {e }}$ siècle. L'économie affective qui semble avoir sous-tendu les argumentations des premiers promoteurs de la protection animale présente de nombreuses similitudes avec celle que nous avons examiné ci-dessus. Quelques différences pourtant méritent sans doute d'être relevées afin de tenter de rendre compte des inflexions les plus récentes. En tout premier lieu, les attitudes précautionneuses à l'égard de l'animal ont longtemps été l'apanage exclusif des classes les plus hautes se percevant comme des happy few doués « d'une sensibilité [qui] n'appart[enait] qu'au petit nombre $»^{35}$. Aujourd'hui, la grande majorité des individus, y compris parmi les franges les plus populaires - et sans doute du fait de l'impact fondamental du média audiovisuel — partagent la même aptitude à s'émouvoir pour le sort des animaux. En second lieu, les préoccupations des premiers protectionnistes concernaient essentiellement les espèces domestiques que la proximité avec l'homme éloignait d'un monde « sauvage » suscitant, pour sa part, bien peu de compassion. De nos jours, la considération et le respect entendent être étendus à tous les êtres vivants y compris aux « bêtes sauvages » qui semblent, d'ailleurs, ne plus en être vraiment. Les évolutions des mœurs décrites par Norbert Élias ont si profondément modifié notre rapport au monde animal que la « sauvagerie » menaçante n'est plus désormais qu'un attribut infamant réservé à des ... hommes mal civilisés.

35 Propos relevé par Valentin Pelosse dans l'un des mémoires étudiés. Bien d'autres éléments cités par l'auteur laissent penser qu'à l'époque la sensibilité à l'égard de la souffrance animale est un signe distinctif que les classes les plus « hautes » opposent à la cruauté à l'égard des animaux dont étaient accusés les classes populaires. 


\section{Références des documents cités.}

d1) Claire Starozinski, La mort donnée en spectacle ou Petit manuel à l'usage des anti-corrida, Alliance pour la Suppression Absolue et Continue des Corridas, Nîmes, 1998, 135 pages.

d2) Claire Starozinski, On est toujours le taureau de quelqu’un, édité par l'auteur, 2003, 91 pages.

d3) Citations du comité d'honneur de l'Alliance pour la suppression des corridas dans On est toujours le taureau de quelqu'un, op.cit.

d4) « Pourquoi l'alliance ? », texte présentant l'Alliance pour la suppression des corridas. Disponible sur le site web de l'Alliance à l'adresse www.anticorrida.org .

d5) « Un combat loyal ? » texte non signé présenté sur le site web de l'Alliance pour la suppression des corridas.

d6) Texte présentant la Fédération de liaisons anti-corrida. (FLAC). Disponible, comme les autres textes des membres de la FLAC, à l'adresse www.anti-corrida.free.fr.

d7) Clavijo (R.) et Lassale (G.) « La corrida négation des droits de l'animal » (FLAC).

d8) Joron (A.) et Querelle (J.), « Les Écoles Taurines ou l'initiation au sadisme » (FLAC).

d9) Camisuli (A.), «Commission éthique et historique » et Querelle (J.), « Motivations des spectateurs de corridas » (FLAC).

d10) Joron (A.), « Elevage de taureaux dits 'de combat'» (FLAC).

d11) Garrigues (J.-P.), « Commission éducation. Réseau de vigilance pédagogique contre la violence tauromachique » (FLAC).

d12) Querelle (J.) et Lassale (G.), « Hommage au Professeur Théodore Monod» (FLAC).

d13) Page (D.), La tauromachie, manuel apologétique à destination des publics néophytes, Éditions Sud-Ouest, Bordeaux, 1993. 
Aficionados et opposants à la tauromachie : les formes plurielles de la civilisation.

Christophe TRAÏNI

L'opposition à la tauromachie espagnole constitue incontestablement un cas emblématique des engagements visant à édifier la cause animale. A partir de la théorie de Norbert Elias relative aux processus de civilisation, l'auteur s'efforce de mettre en exergue les économies émotionnelles qui sous-tendent aussi bien la passion des aficionados que les dénonciations virulentes des opposants à la corrida. La vocation comparative que le sociologue allemand a toujours attribuée à sa théorie est rappelée ici avec force afin de rendre intelligible les incompréhensions qui peuvent résulter de différentes modalités d'investissement des valeurs de la civilisation. Cette perspective permet, plus particulièrement, d'expliciter pourquoi et comment le taureau se voit attribué des vertus et un statut des plus divergents.

Aficionados and Opponents to the Tauromachy: the Plural Forms of Civilization. Christophe TRAÏNI

The opposition to the Spanish tauromachy constitutes an emblematic case of involvement aiming to build up the animal cause. Starting from Norbert Elias theory concerning the civilisation processes, the author attempts to point out the emotional economies which underlie both aficionados' passion and the denunciations of the opponents to the corrida. The author refers here to the comparative vocation of Elias theory to make intelligible the incomprehension which may result from the different ways of investing the civilisation values. This perspective allows explicating why and how the bull takes on different virtues and statutes.

Christophe TRAÏNI est maître de conférences en science politique à l'Institut d'Etudes Politiques d'Aix-en-Provence et est rattaché au Centre de Science Politique Comparative (CSPC). Il a consacré sa thèse de doctorat à l'étude des transformations des formes d'engagement à partir de terrains d'enquête français et italiens. Il a publié Les braconniers de la République. Les conflits autour des représentations de la Nature et la politique, Presses Universitaires de France, janvier 2003. "Territoires de chasse», Ethnologie française, Presses Universitaires de France, janvier 2004. "Arles, loin de la scène politique nationale », dans Dolez B. et Laurent A. (dir.), Le vote des villes. Les élections municipales des 11 et 18 mars 2001, Paris, Presses de Sciences Po, mars 2002. "Les sociabilités musicales induisent-elles de nouveaux répertoires de prise de parole publique ?», publié in François B. et Neveu E. (dir.), Espaces publics mosaïques. Acteurs, arènes et rhétoriques, des débats publics contemporains, Presses Universitaires de Rennes, 1999. 